\title{
Experimental Modeling of the Formation of the Metallic Core of the Moon by the Method of High-Temperature Centrifuges
}

\author{
Lebedev Evgeny Borisovich", ${ }^{1,}$ Averin Viacheslav Vasilyevich², Lukanin Oleg Alexandrovich² \\ ${ }^{1}$ Vernadsky Institute of Geochemistry and Analytical Chemistry, Russian Academy of Sciences, Moscow, Russia \\ ${ }^{2}$ Baikov Institute of Metallurgy and Material Science, Russian Academy of Sciences, Moscow, Russia
}

Email address:

leb@geokhi.ru (L. E. Borisovich)

${ }^{*}$ Corresponding author

\section{To cite this article:}

Lebedev Evgeny Borisovich, Averin Viacheslav Vasilyevich, Lukanin Oleg Alexandrovich. Experimental Modeling of the Formation of the Metallic Core of the Moon by the Method of High-Temperature Centrifuges. Science Journal of Analytical Chemistry.

Vol. 7, No. 3, 2019, pp. 72-75. doi: 10.11648/j.sjac.20190703.12

Received: June 23, 2019; Accepted: July 27, 2019; Published: August 13, 2019

\begin{abstract}
At early stages of the formation of planetary bodies, silicate, metallic, and sulfide phases, which have been formed in the course of the partial or complete fusion of the initial planetary substance, were subject to a gravitational differentiation. The latter is related to the separation of the crystalline and molten phases in density and composition. Althose in recent years a number of papers deal with the experimental and theoretical determination of the processes of the percalation of metallic melts through the crystalline silicate matrix and the physical properties of the metallic and sulfide melts. For the Moon, the problem of the existence of the metallic or sulfide core still remains unsolved. There is stil the nessity of further investigation in this direction. The possible origin of the Moon's metallic core at the precipitation of iron-sulfide phases during the partial melting of ultramafic material under various redox conditions was experimentally modeled by partially melting the model system olivine (85 wt \%)+ ferrobasalt $(10 \mathrm{wt} \%)+$ metallic phase $\mathrm{Fe}_{95} \mathrm{~S}_{5}(5 \mathrm{wt} \%)$ in a high-temperature centrifuge at $1430-1450^{\circ} \mathrm{C}$.
\end{abstract}

Keywords: Origin of the Moon, Experiment, High-Temperature Centrifugation, Partial Melting, Iron Fractionation, Metallic Core

\section{Introduction}

The number of the papers deal with the experimental and theoretical determination of the processes of the percolation of metallic melts through the crystalline silicate matrix and the physical properties of the metallic and sulfide melts, there is still the necessity of further investigation in this direction.

It includes the concentration, thermal and phase convection, the mechanisms of which are thought to be rather intricate A. P. Vinogradov, 1975 [1].

A possible result of these processes is the formation of cores and external silicate shells of terrestrial planets and the Moon. For the Moon, the problem of the existence of the metallic or sulfide core still remains unsolved (Its solution is crucial for understanding the mechanism of moon formation in General and its evolution [2, 3]
Although in recent years a number of papers deal with the experimental and theoretical determination of the processes of the percolation of metallic melts through the crystalline silicate matrix. There is still the necessity of further investigation. [4-6]

These are a number of papers deal with the influence of the deformation on segregation of phases $[8,9]$.

According to E. M. Galimov's model for the origin of the Earth-Moon system [2,6], the Moon was formed of the material of originally chondritic composition during the fragmentation of a dust accumulation, from which both the Moon and the proto-Earth were produced.

Data obtained by experimentally modeling the segregation and accumulation of iron-sulfide melt phases in hightemperature centrifuges show that the percolation of the 
metallic phases through silicate matrix in partial melting zones depends not only on the degree of partial melting (temperature) and the metal content but also on the composition of the equilibrium phases of the system [5], with these parameters notably affecting the rheology of silicate and metallic melts and their interaction with one another and the crystalline phases of the silicate matrix (wettability, viscosity, surface tension, electrical conductivity, elastic wave velocity etc).

\section{Simulation Method}

The segregation of the iron-sulfide metallic phase during partial melting was modeled by means of high temperature centrifugation $[5,12]$.

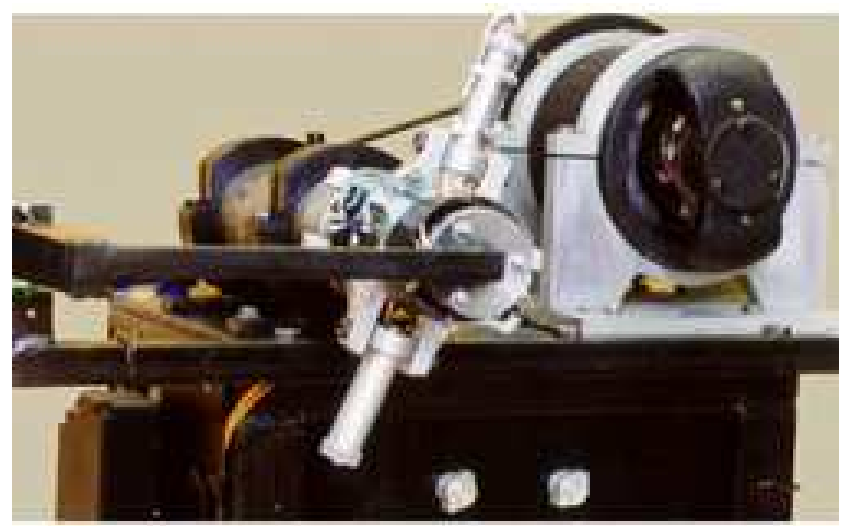

Figure 1. High-temperature centrifuge.

The experimental setup was a centrifuge (3000-6000 $\mathrm{rpm})$ of original design equipped with a heater $[5,12]$. The container with the sample was $\sim 1 \mathrm{~cm}$ long, and the rotation radius was $11 \mathrm{~cm}$. The setup is able to imitate 2000 to $4000 \mathrm{~g}$ gravitation, and the heater maintains an even temperature field throughout the whole sample length at experimental temperatures of $1430-1450{ }^{\circ} \mathrm{C}$. Different redox conditions during the experiments wereensured by various materials around the sample: they contained various metals (iron-based alloys, tungsten, etc.), graphite, and other carbon-bearing materials. Oxygen fugacity $\lg f \mathrm{O}_{2}$ at any given temperature was evaluated from the composition of the quenched phases after the experiments, using three different methodological approaches, which will be described in more detail below.(Lebedev. Kadik, "High temperature centrifuge," RF Patent no. 2082786, Byull. Isobret. No. 18, (1997)).

\subsection{Experimental}

The segregation of the iron-sulfide metallic phase during partial melting was modeled by means of high temperature centrifugation $[5,12]$. The experimental setup was a centrifuge (3000-6000 rpm) of original design equipped with a heater. The container with the sample was $\sim 1 \mathrm{~cm}$ long, and the rotation radius was $11 \mathrm{~cm}$. The setup is able to imitate 2000 to $4000 \mathrm{~g}$ gravitation, and the heater maintains an even temperature field throughout the whole sample length at experimental temperatures of $1430-1450^{\circ} \mathrm{C}$. Different redox conditions during the experiments were ensured by various materials around the sample: they contained various metals (iron-based alloys, tungsten, etc.), graphite, and other carbonbearing materials.

Oxygen fugacity $\mathrm{fO}_{2}$ at any given temperature was evaluated from the composition of the quenched phases after the experiments, using three different methodological approaches, which will be described in more detail below.

The vertical cross sections of the samples obtained after centrifugation and quenching in the experiments of CS-49, CS-122, CS-105, CS-96, CS-110, CS-41 and the shape of the metal drop in the samples in the experiments of CS-49, CS65, CS-96, CS-97, CS-105, CS-122 and in the experiments, in addition to the pure effect of centrifugal forces on the melts and as a result of the additional load and the movement of the melt in the lower pressure zone are shown in Figure 2, Figure 3, and the compositions of silicate melts after the experiments (wt. \%) Table 1.

Table 1. The compositions of silicate melts after the experiments (wt. \%).

\begin{tabular}{|c|c|c|c|c|c|c|c|c|c|c|}
\hline Experiment numbers & $\mathrm{SiO}_{2}$ & MgO & FeO & $\mathbf{A l}_{2} \mathbf{O}_{3}$ & $\mathrm{CaO}$ & $\mathrm{TiO}_{2}$ & $\mathrm{MnO}$ & $\mathrm{Na}_{2} \mathrm{O}$ & $\mathbf{K}_{2} \mathbf{O}$ & $\Sigma$ \\
\hline CS-122 & 50.96 & 10.97 & 9.60 & 13.46 & 10.65 & 0.65 & 0.00 & 1.49 & 0.34 & 98.12 \\
\hline CS-44 & 60.51 & 7.22 & 13.13 & 11.53 & 0.60 & 0.00 & 0.00 & 1.26 & 0.17 & 101.89 \\
\hline CS-105 & 58.65 & 6.35 & 6.45 & 15.27 & 9.25 & 0.80 & 0.37 & 2.30 & 0.74 & 100.18 \\
\hline CS-45 & 62.21 & 17.99 & 4.32 & 7.85 & 5.28 & 0.31 & 0.00 & 0.91 & 0.14 & 99.02 \\
\hline CS-96 & 47.48 & 18.15 & 0.76 & 15.86 & 12.69 & 0.85 & 0.00 & 2.11 & 1.06 & 98.97 \\
\hline CS-57 & 60.81 & 10.89 & 0.40 & 14.79 & 13.23 & 0.37 & 0.20 & 0.60 & 0.10 & 101.38 \\
\hline CS-110 & 60.24 & 17.29 & 0.17 & 12.20 & 7.68 & 0.40 & 0.00 & 0.82 & 0.34 & 99.14 \\
\hline CS-41 & 45.425 & 56.87 & 0.57 & 0.15 & 9.22 & 0.00 & 0.08 & 0.04 & 0.00 & 103.61 \\
\hline CS-49 & 60.31 & 5.31 & 9.50 & 11.18 & 9.80 & 0.25 & 0.26 & 0.89 & 0.97 & 98.65 \\
\hline
\end{tabular}



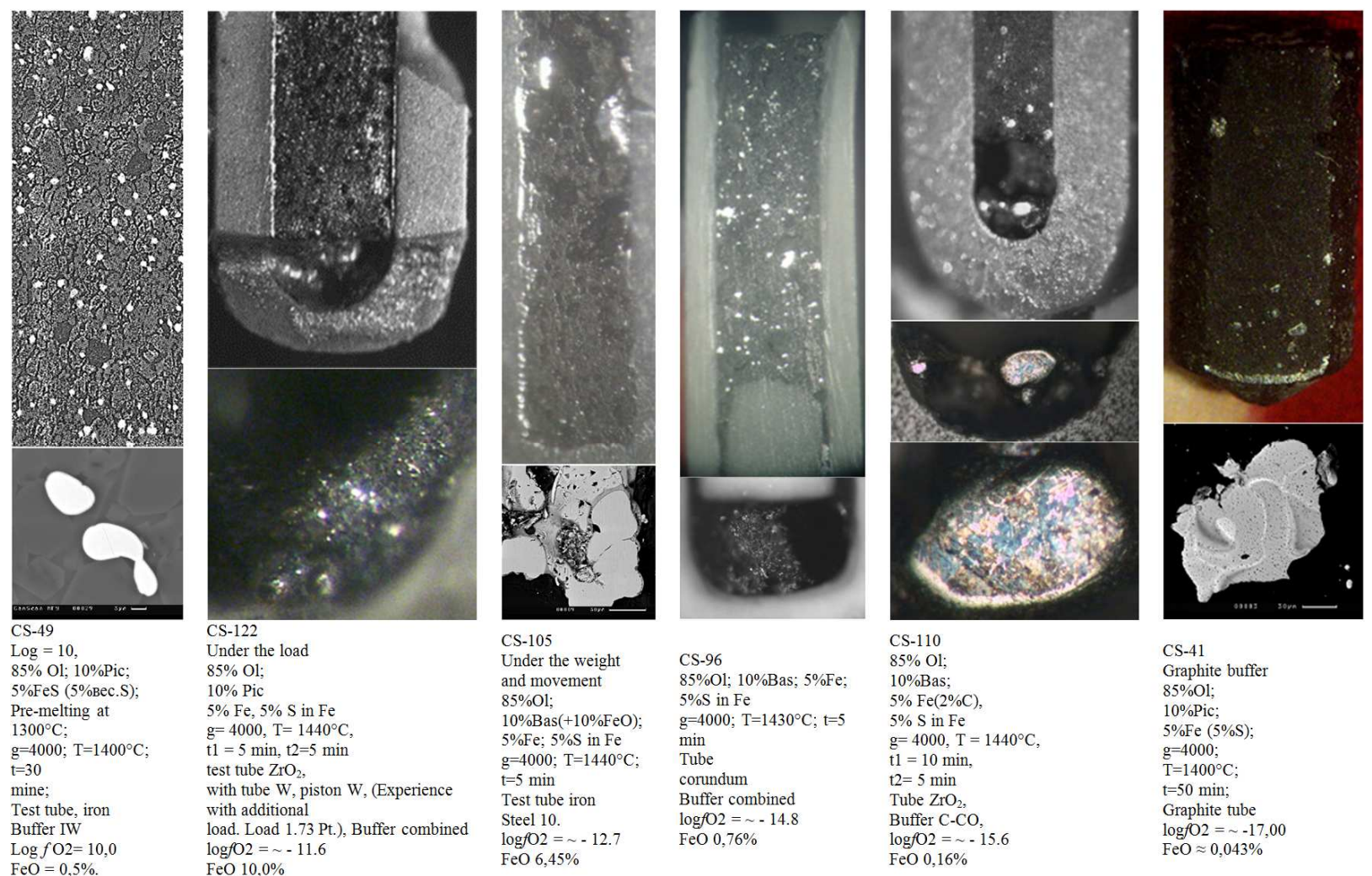

Experience CS-122 with an additional load of $1.73 \mathrm{~g}$ placed on a tungsten piston, which exerted additional pressure on the sample in a tungsten shell.

Figure 2. Distribution of phases after centrifugation in runs CS-49, CS-122, CS-105, CS-96, CS-110, CS-41.
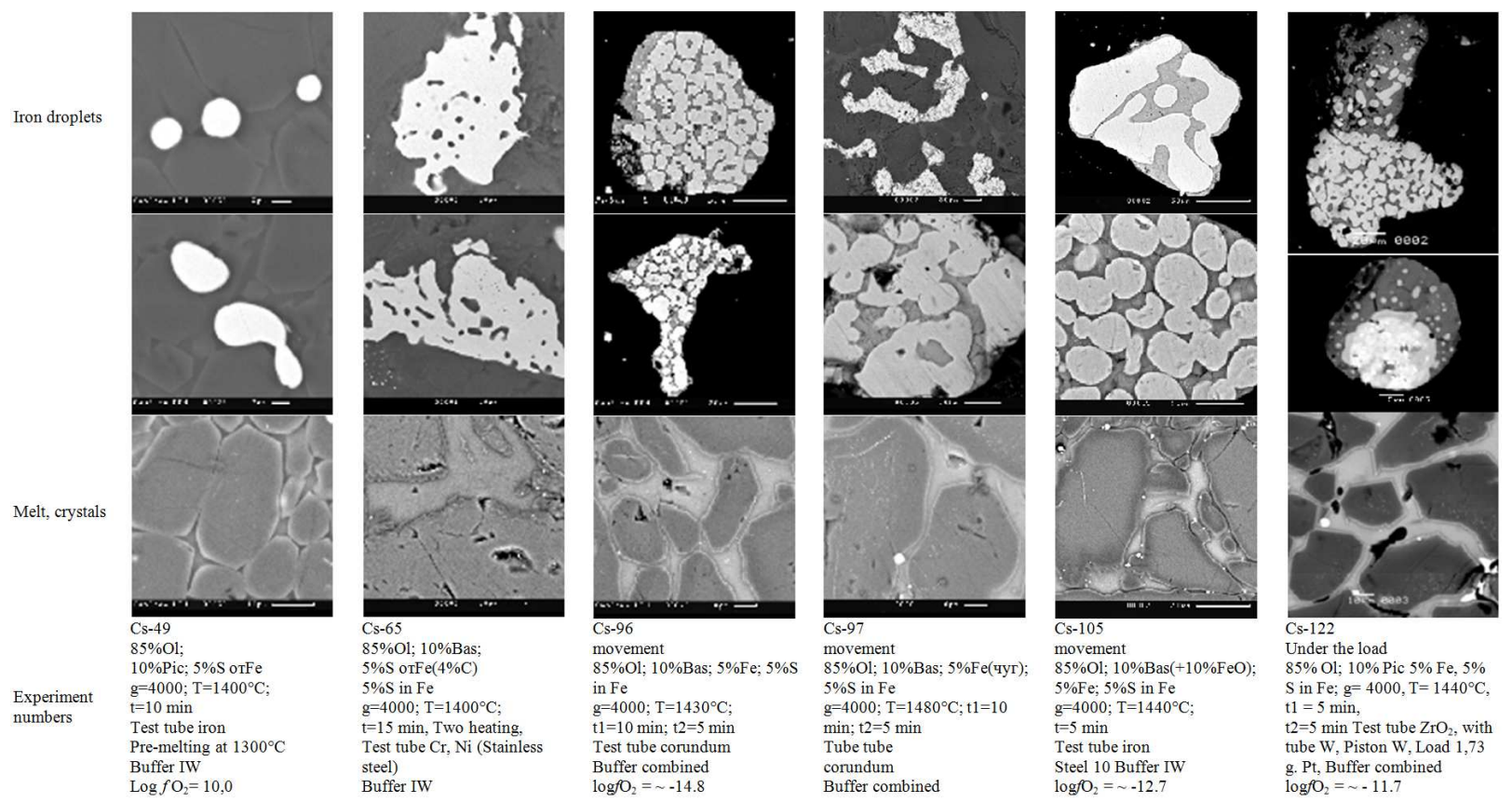

Figure 3. Phase distribution (iron droplets) after centrifugation, works in CS-49, CS-65, CS-96, CS-97, CS-105, CS-122.

\section{Redox Conditions}

The oxygen fugacity in the system whose iron-sulfide melt was in equilibrium with a metal phase can be calculated using the reaction

$$
(\mathrm{FeO})_{\mathrm{L}}=(\mathrm{Fe})_{\text {Met. }}+\mathrm{O}_{2}
$$

The oxygen fugacity $f \mathrm{O}_{2}$ was determined from the composition of the quenched experimental silicate melts (glasses). A decrease in $f \mathrm{O}_{2}$ is proved to be favorable for the segregation of iron-sulfide melt from the silicate matrix. The 
metallic phase is most effectively segregated in the form of melt droplets, and these droplets are accumulated in the lower portions of the samples under strongly reduced conditions, at $f \mathrm{O}_{2} \sim-5.5(\mathrm{CS}-110)$ orders of magnitude lower than the iron-wustite buffer [10].

\section{Discussion}

Calculations show that the observed distribution of siderophilic elements on the Moon can be achieved if the iron is separated from the initial material of the chondrite composition under the condition of its partial melting. It is believed that a lower degree of partial melting prevents the metal from seeping into the core.

The possibility of percolation of iron-rich metal melts through the crystal matrix of ultrabasic composition, which is in a partially molten state, depends mainly on the energies of the interphase interaction of the crystalline and molten phases in the system, which determine the following physical properties of phases as wetting, surface and interphase tension of coexisting melts and crystals. The criterion of melt seepage through the crystal matrix is the value of the dihedral wetting angle $(\Theta)[10]$.

At low degrees of melting and small amounts of iron and sulfur in the deformation of the silicate frame and flow in the lower pressure zone gives sufficient justification to one of the possible mechanisms of formation of theMoon core percolation model (seepage and deposition) in contrast to the mega-impact (shock) model orformation and evolution of a lunar core from ilmenite-rich magma ocean cumulates using a thermo-chemical convection model [11].

\section{Conclusions}

1. The method of high-temperature centrifugation was used for experimental modeling of iron segregation under partial melting of a model ultrabasic substance under certain redox conditions.

2. The oxygen fugacity in the experiments was estimated by the composition of the silicate melt in the quenching samples after the experiments using three thermodynamic methods.

3. In experiments $\lg f \mathrm{O} 2$ varied from $\sim 2$ to $\sim 5.5 \log$. units below the IW buffer.

4. The most complete deposition of the iron melt is observed in the experience of CS-110 to clean the centrifugal field with the fugacity of oxygen $\lg f \mathrm{O}_{2}=\sim-15.6$ in residual amounts in the silicate melt $\mathrm{FeO}=0,16 \%$.

5. The result obtained indicates the possibility of the implementation of the proposed mechanism of formation of the core of the Moon from a primary substance, similar to the composition of CI carbonaceous chondrites.

\section{Acknowledgements}

This study was financially supported by the Russian
Foundation for Basic Research (project no. 07-05-00630) and the Program for Fundamental Research under the presidium of the Russian Academy of Sciences (Grant 24).

*E. B. Lebedev, A. A. Kadik, E. M. Galimov. Segregation of molten metal through partially molten silicate: simulation using a high temperature centrifuge. 32nd IGC - Florence, 2004; "T06.02

\section{References}

[1] A. P. Vinogradov,"Differentiation of the lunar and planetary material into shells," inCosmochemistry of the Moon and Planets, Ed. by A. P. Vinogradov, (Nauka, Moscow, 1975), p. 5-28.

[2] E. M. Galimov, "Problem of the Moon origin," in Main Directions in Geochemistry. On100th A. P. Vinogradov Anniversary, Ed. by E. M. Galimov (Nauka, Moscow, 1995), pp. 8-43. in Russian].

[3] S. K. Runcorn, "The formation of the lunar core, " Geochim. Cosmochim. Acta. 60, 1205- 1208 (1996).

[4] E. B. Lebedev and E. M. Galimov, "Experimental modeling of the origin of the Moon's metallic core at partial melting," Geochem. Int. 50 (8), 639-648 (2012).

[5] E. B. Lebedev, A. A. Kadik, O. L. Kuskov, A. M. Dorfman, and O. A. Lukanin "Themotion of sulfide phases in a partially molten silicate material: application to the problem ofthe formation of planetary cores, " Solar Syst. Res. 33 (5)346-355 (1999).

[6] E. M. Galimov and A. M. Krivtsov, Origin of the Moon. New Concept. Geochemistry and Dynamics (De Gruyter \& Co. KG, Berlin-Boston, 2013).

[7] E. B. Lebedev and E. M. Galimov, "Physicochemical conditions of experimental modeling of the formation of the Moon's metallic core at partial melting, " in Problems of Origin and Evolution of Biosphere, Ed. By E. M. Galimov, (KRAS AND, Moscow, 2013), pp. 183-193 [in Russian].

[8] M. C. Shannon and C. B. Agee, "Percolation of core melts at lower mantle conditions," Science 280, 1059-1061 (1998).

[9] T. Rushmer, et al. "Fe-liquid segregationin deforming planetosimals: Coupling core-forming compositions with transport phenomena," Earth Planet. Sci. Lett. 239, 185-202 (2005).

[10] E. B. Lebedev, V. V. Averin, O. A. Lukanin, I. A. Roshchin, N. N. Kononkova, and E. A. Zevakin. Effect of Redox Conditions on Iron Metal Phase Segregation the during Experimental High-Temperature Centrifuge Modeling of the Origin of the Moon's CoreGeochem. Int. 2016, Vol. 54, No. 7, pp. 609-617.

[11] Jellie de Vriesaet al. Formation and evolution of a lunar core from ilmenite-rich magma ocean cumulates. Earth and Planetary Science Letters 292 (2010)139-147.

[12] A. A. Kadik and E. B. Lebedev, "Differentiation of partially molten zones of the Moon under conditions of multiphase flow of deep-seated material: a high-temperature centrifuge simulation," Solar Syst. Res. 33 (5), 392-399 (1999). 